\title{
Burnout and depression among Spanish periodontology practitioners
}

\author{
Jose-Vicente Ríos-Santos ${ }^{1}$, Mercedes Reyes-Torres ${ }^{1}$, Ana López-Jiménez ${ }^{2}$, Juan-Manuel Morillo-Velázquez ${ }^{1}$, \\ Pedro Bullón ${ }^{1}$
}

${ }^{1}$ Department of Stomatology and Periodontology

${ }^{2}$ Department of Experimental Psychology University of Seville (Spain)

\author{
Correspondence \\ Facultad de Odontología \\ $C /$ Avicena, $s / n$ \\ 41009 Sevilla (Spain) \\ jvrios@us.es
}

Received: 08/09/2009

Accepted: 24/12/2009

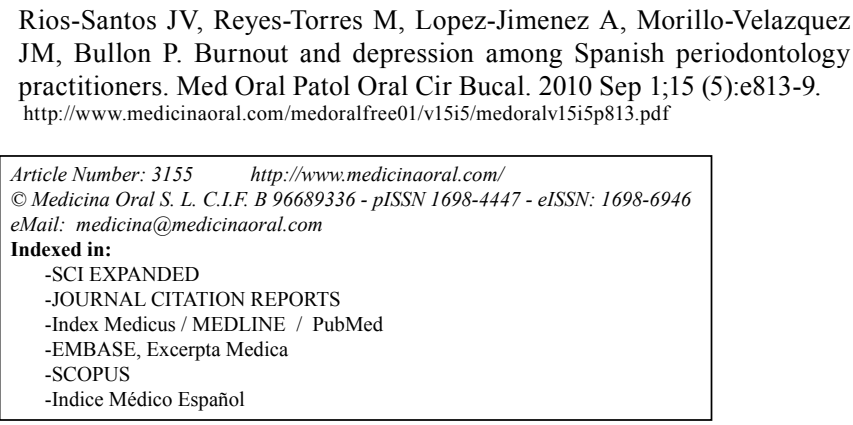

\begin{abstract}
Objectives: The objective of this study was to determine the prevalence of burnout syndrome and depression symptoms among periodontology practitioners, as well as the relationship with socio-demographic variables and work stress.

Study Design: A total of 284 subjects, members of the Spanish Society of Periodontology (SEPA), were selected for this study. The Spanish version of the Maslach Burnout Inventory (MBI) and the Tetradimensional Estructural Questionnaire for Depression (TEC-DE) were sent by post to all participants. Descriptive statistics in addition to mean comparison and chi-square test were used for data analysis.

Results: A response rate of $59.85 \%$ was obtained. From the total sample, $40 \%$ shows high levels of emotional exhaustion, $20 \%$ increased depersonalization and $11.20 \%$ diminished personal accomplishment. The prevalence of any depression symptom was nearly $16 \%$. Moreover, higher prevalence was found among those practitioners not working a continuous shift and not practicing periodontology on an exclusive basis.

Conclusion: Among Spanish periodontology practitioners, a high prevalence of burnout and depression exists.
\end{abstract}

Key words: Burnout, depression, periodontology.

\section{Introduction}

Currently, work health is considered a critical factor in developed countries (1). Some occupations are generally known as powerful stress inducers, and those related to health care are included. This exposure to stress inducers may cause disorders as burnout syndrome and depressive symptoms.

The term burnout was introduced in the 1970s by Maslach and Leiter (2), and was defined as a syn- drome with emotional exhaustion, depersonalization and diminished personal accomplishment that can occur among individuals working with people. Physiological, psychological and behavioral dimensions are affected by burnout, and several symptoms can subsequently occur (feelings of loneliness, apathy, mood disorders, hypertension, fatigue, etc), with negative consequences for the working environment and the own health status. Currently, depression is considered the most frequent 
psychiatric disorder, and the second most relevant behind schizophrenia. Depression is an affective disorder characterized by a very low mood and an inability to experience pleasure. Some reports found a higher incidence of depressive symptoms in health care providers (3), although it is not sufficiently clear that work stress existing in these occupations may be a potential cause. Periodontology is an increasingly important speciality in Europe. In order to promote standardization of postgraduate education in Europe, the European Federation of Periodontology (EFP) was founded in 1990, being Spain one of its founder members (4). In this country, the Spanish Society of Periodontology and Osteointegration (SEPA) is the national periodontal scientific society that encompasses the vast majority of periodontal practitioners. To our knowledge, there is no previous report about the burnout syndrome and depression in this dental speciality. Therefore, our aim has been to analyze the psychological well-being of Spanish periodontology practitioners regarding their work.

\section{Material and Methods}

\section{Study population}

All members of the SEPA were selected as study population for this research $(\mathrm{N}=1,419$ in November, 2004). By using a stratified random sampling with proportional allocation by region, a final sample of 284 subjects was obtained in order to include $20 \%$ of the members.

\section{Instruments}

A cover letter outlining the main characteristics of the study, in addition to several instruments for data collection, was sent to selected participants by post. A questionnaire was developed for collecting demographic variables: age, gender, academic graduate (only dentist or dentist with a graduate in medicine), marital status, date of graduation, number of years working with patients, health sector (public, private or both), type of activity if working in the private health sector (partnership, freelance or employee), working hours per week, free days per week, type of working shift (continuous or split), and type of member in SEPA (specialist or other).

Maslasch et al. Burnout Inventory (MBI) (2) was the instrument chosen for assessing burnout, because it is the most commonly used in research on this topic, and it achieves good results when using for groups. Other authors have confirmed its validity in a dental population (5). It consists of three scales to measure the three dimensions of burnout: emotional exhaustion, depersonalization and diminished personal accomplishment. In this study, validated score ranges for Spanish general population and physicians were used. On the other hand, three variables representing all dimensions of the syndrome in its low, medium or high grade were created using the 33th and 66th percentiles, so our own score ranges were obtained to determine emotional exhaustion, depersonalization or diminished personal accomplishment in our sample (Table 1). A variable called burnout core was created in order to represent high scores in emotional exhaustion and depersonalization, according to the score ranges used.

The reduced version of the Tetradimensional Estructural Questionnaire for Depression (TEC-DE) was used to determine the prevalence of depression among Spanish periodontics practitioners. TEC-DE consists of four parts related to primary depressive symptoms: depressed mood (type I), anergy or energy depletion (type II), communication disorder or lack of sociability (type III), and disturbed biopsychic rhythms (type IV) with disorder of circadian rhythm. This instrument may categorize depressive syndrome according to the number of affected dimensions as uni-, bi-, tri- or tetradimensional pictures. In this study, each dimension was first

Table 1. Score ranges used to estimate the prevalence of burnout syndrome in the tested population. DP, depersonalization; EE, emotional exhaustion; PA, personal accomplishment.

\begin{tabular}{|c|c|c|c|c|}
\hline & Dimension & Low & Medium & High \\
\hline \multirow{3}{*}{$\begin{array}{l}\text { Spanish general popula- } \\
\text { tion }\end{array}$} & $\mathrm{EE}$ & $<15$ & $15-24$ & $>24$ \\
\hline & DP & $<4$ & 4- 9 & $>9$ \\
\hline & PA & $<33$ & $33-39$ & $>39$ \\
\hline \multirow{3}{*}{ Spanish physicians } & $\mathrm{EE}$ & $<22$ & $22-31$ & $>31$ \\
\hline & DP & $<7$ & $7-13$ & $>13$ \\
\hline & $\mathrm{PA}$ & $<30$ & $30-35$ & $>35$ \\
\hline \multirow{3}{*}{$\begin{array}{l}33^{\text {th }}-66^{\text {th }} \text { percentiles from } \\
\text { our SEPA sample }\end{array}$} & $\mathrm{EE}$ & $<15$ & $15-26$ & $>26$ \\
\hline & DP & $<4$ & 4- 9 & $>9$ \\
\hline & $\mathrm{PA}$ & $<41$ & $41-45$ & $>45$ \\
\hline
\end{tabular}


analyzed separately, and subsequently the four types were considered as a whole, defining a variable called total depression.

\section{Procedure}

Each participant received by post an envelope referring from the research center, containing a cover letter by the researcher group, a questionnaire to be completed and its instructions, as well as the identification data. Along with these documents, another smaller pre-stamped envelope contained a form for the answers. Participants had to send these documents by post to a notary collaborating with the research group. This lawyer collected all documents and maintained the anonymity in order to guarantee the confidentiality of responses obtained by participating practitioners, a prerequisite for the reliability of the study. A total of 284 questionnaires were sent. This lawyer communicated the research group which participants sent his/her response, in order to contact the remaining participants by phone.

Statistical analysis

A descriptive analysis was performed to determine the prevalence of burnout syndrome and profiles for the three dimensions of stress and depressive symptoms according to the socio-demographic variables included in this study. In addition, comparison of means and chi-square tests were used to determine the relationship between the prevalence of burnout and depressive symptoms and the various socio-demographic and work variables. The level of statistical significance was established at $\mathrm{p}<0.05$. Statistical software package SPSS version 14.0 was used for data processing.

\section{Results}

The total number of responders was 170 , representing a response rate of $59.85 \%$. (Fig. 1) summarizes data about the distribution of the sample according to socio-demographic and work variables.

(Table 2) represents the categorization of participants according to obtained scores for each dimension of burnout, by applying the corresponding score ranges. According to the score range for Spanish population, $40 \%$ of dentists show increased emotional exhaustion, whereas only $20 \%$ present increased depersonalization and $11.2 \%$ show diminished personal accomplishment. According to the score range for our own sample, similar results have been obtained. Thus, $13.84 \%$ suffer from the three dimensions of burnout syndrome. The prevalence of "burnout core" varied according the score range used, being lower for that obtained from Spanish physicians (3.5\% vs. 12.9 and $11.8 \%$, respectively).

Scores for each dimension according to socio-demographic and work variables are shown in (Table 3). Significant gender differences were found for the three dimensions. Hence, men showed increased emotional exhaustion and depersonalization $(\mathrm{p}<0.05)$, as well as diminished personal accomplishment $(\mathrm{p}<0.001)$. Moreover, significantly increased emotional exhaustion was found among dentists with a graduate in medicine $(\mathrm{p}<$ 0.05). In the same way, not being a specialist member was significantly associated with diminished personal accomplishment $(\mathrm{p}<0.05)$. Most dentists working 16 to 22 years showed increased emotional exhaustion, but also higher levels of personal accomplishment.

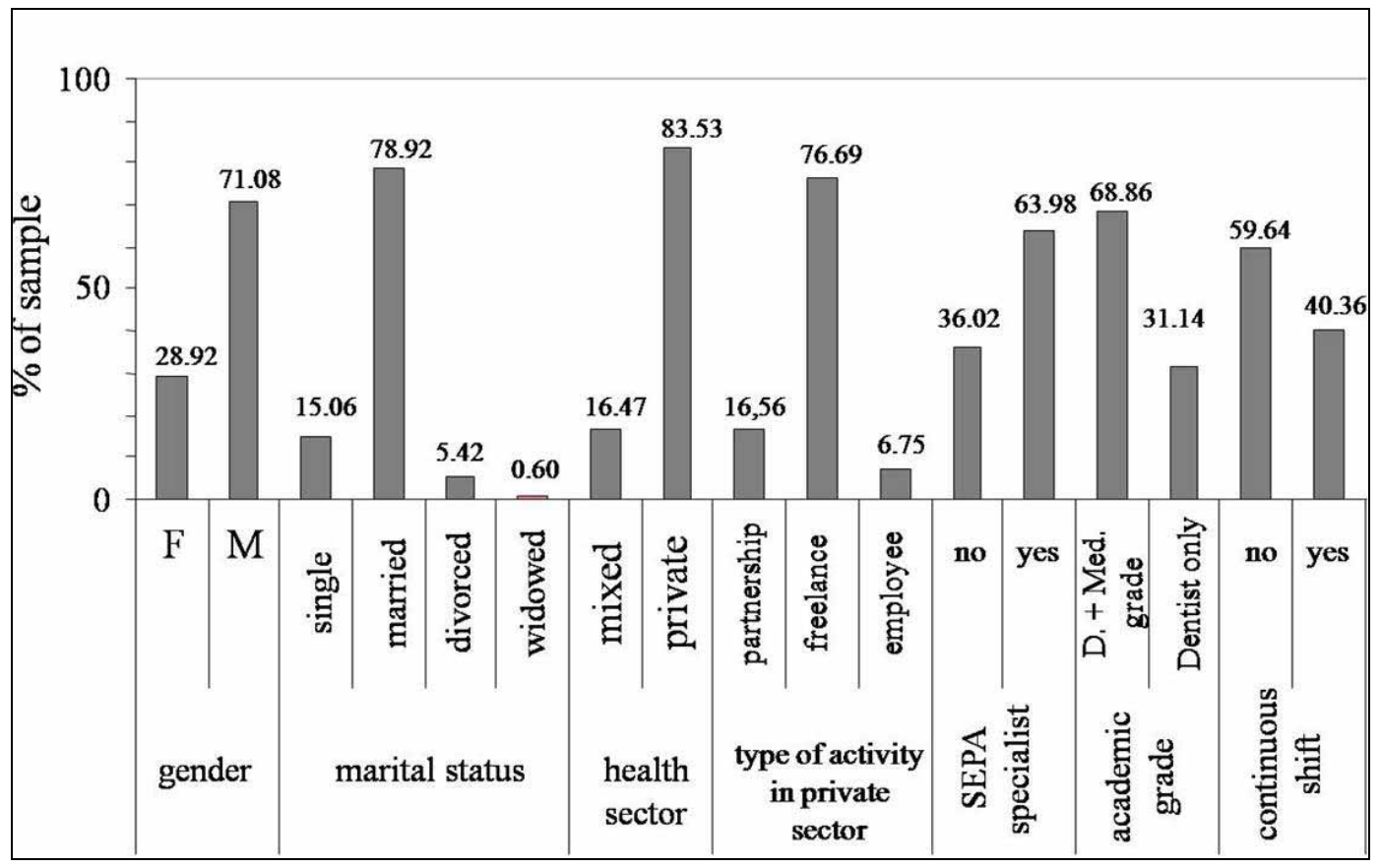

Fig. 1. Distribution of socio-demographic and work variables. 
Table 2. Distribution of burnout dimensions according to several score ranges.

\begin{tabular}{|c|c|c|c|c|}
\hline $\begin{array}{l}\text { Reference } \\
\text { population }\end{array}$ & Dimension & Score & $\mathbf{N}$ & $\begin{array}{l}\text { Prevalence } \\
\text { (\%) }\end{array}$ \\
\hline \multirow{9}{*}{$\begin{array}{l}\text { Spanish } \\
\text { general } \\
\text { population }\end{array}$} & \multirow{3}{*}{ emotional exhaustion } & Low & 55 & $32.4 \%$ \\
\hline & & medium & 47 & $27.6 \%$ \\
\hline & & high & 68 & $40.0 \%$ \\
\hline & \multirow{3}{*}{ depersonalization } & Low & 71 & $41.8 \%$ \\
\hline & & medium & 65 & $38.2 \%$ \\
\hline & & high & 34 & $20.0 \%$ \\
\hline & \multirow{3}{*}{ personal accomplishment } & Low & 121 & $71.2 \%$ \\
\hline & & medium & 30 & $17.6 \%$ \\
\hline & & high & 19 & $11.2 \%$ \\
\hline \multirow{9}{*}{$\begin{array}{l}\text { Spanish } \\
\text { physicians }\end{array}$} & \multirow{3}{*}{ emotional exhaustion } & Low & 84 & $49.4 \%$ \\
\hline & & medium & 38 & $22.4 \%$ \\
\hline & & high & 48 & $28.2 \%$ \\
\hline & \multirow{3}{*}{ depersonalization } & Low & 113 & $66.5 \%$ \\
\hline & & medium & 44 & $25.9 \%$ \\
\hline & & high & 13 & $7.6 \%$ \\
\hline & \multirow{3}{*}{ personal accomplishment } & Low & 147 & $86.5 \%$ \\
\hline & & medium & 9 & $5.3 \%$ \\
\hline & & high & 14 & $8.2 \%$ \\
\hline
\end{tabular}

Table 3. Distribution of burnout dimensions according to several socio-demographic variables. Values are expressed as mean \pm standard deviation.

\begin{tabular}{|l|c|c|c|c|}
\hline \multicolumn{1}{|c|}{ Variable } & Eomen & $\begin{array}{c}\text { Emotional } \\
\text { exhaustion }\end{array}$ & Depersonalization & $\begin{array}{c}\text { Personal } \\
\text { accomplishment }\end{array}$ \\
\hline \multirow{2}{*}{ Gender } & Men & $22.63 \pm 12.31$ & $5.52 \pm 4.71$ & $43.53 \pm 4.63^{\dagger}$ \\
\cline { 2 - 5 } & Only dentist & $18.60 \pm 11.54^{*}$ & $5.26 \pm 4.34$ & $42.28 \pm 5.34$ \\
\cline { 2 - 5 } Academic grade & $\begin{array}{c}\text { Dentist with a } \\
\text { grade in medicine }\end{array}$ & $22.91 \pm 11.55$ & $5.58 \pm 4.78$ & $41.01 \pm 6.87$ \\
\hline \multirow{2}{*}{ SEPA specialist } & Yes & $21.27 \pm 11.26$ & $5.36 \pm 4.44$ & $42.24 \pm 6.08^{*}$ \\
\cline { 2 - 5 } & No & $22.58 \pm 12.38$ & $5.51 \pm 5.05$ & $40.00 \pm 7.00$ \\
\hline \multirow{2}{*}{$\begin{array}{l}\text { Working hours } \\
\text { per week }\end{array}$} & $<37 \mathrm{hr}$ & $20.42 \pm 11.42$ & $5.40 \pm 4.49 *$ & $41.67 \pm 6.07$ \\
\cline { 2 - 5 } & $37-40 \mathrm{hr}$ & $19.69 \pm 11.02$ & $4.44 \pm 3.78$ & $41.30 \pm 6.80$ \\
\hline \multirow{2}{*}{ Working shift } & $>40 \mathrm{hr}$ & $24.52 \pm 12.28$ & $6.64 \pm 5.35$ & $41.42 \pm 6.59$ \\
\cline { 2 - 5 } & continuous & $19.01 \pm 10.85^{*}$ & $4.55 \pm 3.92^{*}$ & $43.10 \pm 4.85^{\dagger}$ \\
\cline { 2 - 5 } & split & $23.22 \pm 11.97$ & $6.14 \pm 4.98$ & $40.30 \pm 7.16$ \\
\hline
\end{tabular}

$* \mathrm{p}<0.05$

$\dagger \mathrm{p}<0.01$ 
Dentists simultaneously working in public and private sectors showed increased levels of burnout than those working exclusively in the private health sector. Increased emotional exhaustion was more prevalent among those working as freelance, although employees showed higher levels of depersonalization. Those working as freelance in the private sector showed higher levels of personal accomplishment. Significantly lower scores were obtained for dentists working a continuous shift $(\mathrm{p}<0.05)$. Moreover, increased number of working hours was significantly associated with depersonalization $(\mathrm{p}<0.05)$. Subjects working a split shift showed significantly higher prevalence of burnout core than those working a continuous shift $(19.1 \%$ vs. $3.1 \%$, p < $0.01)$.

With respect to depression, the most prevalent symptoms were depressed mood and anergy (14.3\%). In the bi- and tridimensional analysis, co-occurrence of disturbed biopsychic rhythms and disorder of communication (9\%) and the triad of depressed mood, anergy and disorder of communication (5.3\%) were the most common combinations, respectively. All four symptoms occur in $1.8 \%$ (Fig 2). Mean score for total depression was $25.43 \pm 10.94$.
Most socio-demographic variables didn't show any significant association with depression symptoms. Being a specialist member was significantly associated to clinical categories I-II, III-IV and I-II-IV $(p<0.05)$. With respect to years of work experience, working for 15-22 years was significantly associated with clinical individual categories I and III $(p<0.05)$. Women not working a continuous shift and being a specialist member have lower mean scores compared to those specialists working a continuous shift. By contrast, men not working a continuous shift and being a specialist member reached higher mean scores for depression. Among those that were not specialists, working a continuous shift was associated with lower mean scores for depression.

\section{Discussion}

In the present study, the prevalence of burnout syndrome and depression among Spanish periodontists was determined. SEPA was chosen in our study because this organization consists of both dentists practicing periodontology on an exclusive basis and those who practice it from a generalist perspective.

Response rate in previous reports about burnout in den-

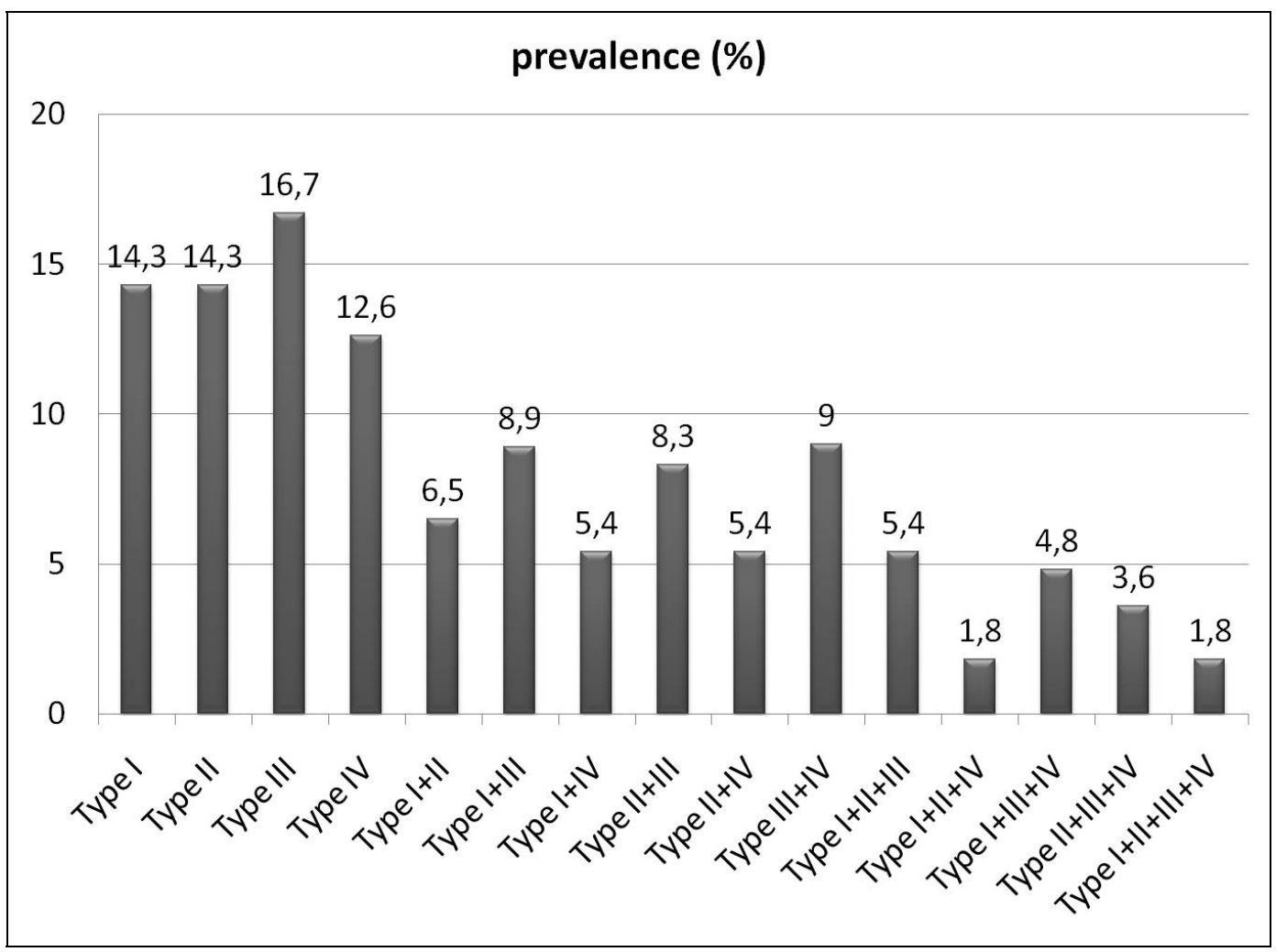

Fig. 2. Prevalence of depressive symptoms among Spanish periodontologists: depressed mood (type I), anergy or energy depletion (type II), communication disorder or lack of sociability (type III), and disturbed biopsychic rhythms (type IV) with disorder of circadian rhythm. 
tal practitioners ranges from $77 \%$ (6) to $53 \%$ (7), so our results (59.85\%) are comparable. With respect to depression, our response rate is slightly lower than previous reports. Thus, Puriene et al. (8) found a response rate of $68.2 \%$ in Lithuania, whereas Ahola et al. (9) reported a rate of $84 \%$ in Finland. However, our study improve the results reported in dentists from Michigan (34.41\%) (10).

Some studies have focused on determining burnout levels among European dental students. In Spain, the prevalence of burnout is low (2-3\%) in a sample from the University of Barcelona (11). Nevertheless, other authors have found higher burnout levels among dental students from other European countries, such as Germany (12), and increasing scores exist in more advanced courses (13), although these levels don't reach those existing in dental practitioners.

With respect to health care providers from other countries, a high emotional exhaustion was reported in 35.8\% of Australian nurses (14). However, they showed lower prevalence of depersonalization compared to our results (14.1\% vs. $20 \%$ ). Diminished personal accomplishment is similar in both studies $(\sim 11 \%)$. With respect to burnout in dental care providers from other countries, the prevalence of increased scores in all three burnout dimensions among Dutch dentists was $21 \%$ (5), slightly higher than ours. However, taking into account each dimension separately, our study shows a higher prevalence of emotional exhaustion, which is considered the central dimension of the syndrome. It is important to highlight that, according to the Spanish population score range, $27 \%$ and $38 \%$ of SEPA members show a medium score in emotional exhaustion and depersonalization, respectively. These data reflect that these subjects likely suffer from these two dimensions in the future. In a recent study from UK (15), Denton found that nearly $8 \%$ of dentists had scores suggestive of burnout on all three scales. In addition to this, $6.7 \%$ had high levels of emotional exhaustion and depersonalization, and $11.8 \%$ had high levels of emotional exhaustion and low levels of personal accomplishment.

The prevalence of depression symptoms in our sample is higher than that in a similar population from USA (7) (9\%), and lower than samples from other European countries, such as Finland (23\%) (10). In other health care providers, such as nurses, higher levels of depression were found (16), with $20 \%$ of nurses suffering from mild depression symptoms, $6.25 \%$ with moderate depression, and $1.6 \%$ with severe depressive symptoms. Hence, depression seems a common disorder among health care providers. In contrast, other European study found the occurrence of depression in $4-5 \%$ of sample consisting of several workers from the service sector (17).

In our study, women show decreased levels of emotional exhaustion and depersonalization, as well as increased levels of personal accomplishment, compared to men. Our results partly agree, therefore, with a previous study conducted among Dutch dentists (18). However, higher levels of depersonalization among women were found in this study. Moreover, these authors describe some differences in the age and working hours according to gender; hence, these factors may explain the occurrence of those differences previously attributed exclusively to gender.

Depression symptoms were more prevalent among males in our sample. In contrast, Dorman et al. (17) found a higher proportion of women in a German sample, a similar result compared to Greek nurses (19), Brazilian oral maxillofacial surgeons (20) and US dentists (7). This report found a significantly higher prevalence of depression among women compared to men, particularly in pediatric dentistry and periodontology practitioners.

As previously mentioned, working in partnership had lower scores in all symptoms, both in burnout and depressive syndrome. This may be explained by the greater sense of security or support when making joint decisions or coping problems, or the tax benefits derived from a partnership. In the previously mentioned report from UK (15), emotional exhaustion was related to the number of hours worked, with those working higher number of hours showing the highest scores on this trait, findings similar to ours.

It is very interesting to highlight the relationship between increased personal accomplishment and being a specialist member, and the lower incidence of depressive symptoms. Another important finding is that continuous shift influences differentially in males and females, in such a manner that women show higher depression scores compared to men. A putative explanation of these data is the incompatibility to familial life in the case of women.

Other relevant data derived from our study is the potential association of depression and burnout with the years of work experience, in such a manner that intermediate stages of work life seem more susceptible to these mental disorders. A possible explanation of this finding might be the loss of motivation as experience is increasing, repetitive tasks and monotony of work shift.

The higher levels of emotional exhaustion among SEPA members are very important. Several reasons can explain this phenomenon. On the one hand, it is very important to take into account the analyzed specialty. Since patient is diagnosed, periodontitis implies a great concern for him/her, with an increased demand for the practitioner, because explanation of the disease and motivation of the patient are essential to managing this condition. Visits to the specialist are very frequent and, in many cases, procedures which may cause doubts, anxi- 
ety and reduced compliance in the patient must be performed in order to manage periodontitis. On the other hand, it is necessary to bear in mind that most members of the sample are periodontology specialists; this implies a great activity in periodontal and implant surgery and implant, with the subsequent increased stress which leads to higher levels of emotional exhaustion.

\section{References}

1. Schaufeli WB, Bakker A. Job demands, job resources, and their relationship with burnout and engagement: a multisample study. J Organiz Behav. 2004;25:293-315.

2. Maslach C, Leiter MP. Early predictors of job burnout and engagement. J Appl Psychol. 2008;93:498-512.

3. Alexander RE. Stress-related suicide by dentists and other health care workers. Fact or folklore? J Am Dent Assoc. 2001;132:786-94.

4. Sanz M, Van der Velden U, Van Steenberghe D, Baehni P. Periodontology as a recognized dental speciality in Europe. J Clin Periodontol. 2006;33:371-5.

5. Gorter RC, Eijkman MA, Hoogstraten J. Burnout and health among Dutch dentists. Eur J Oral Sci. 2000;108:261-7.

6. Gorter RC, Albrecht G, Hoogstraten J, Eijkman MA. Professional burnout among Dutch dentists. Community Dent Oral Epidemiol. 1999;27:109-16.

7. Mathias S, Koerber A, Fadavi S, Punwani I. Specialty and sex as predictors of depression in dentists. J Am Dent Assoc. 2005;136:1388-95.

8. Puriene A, Aleksejuniene J, Petrauskiene J, Balciuniene I, Janulyte V. Self-perceived mental health and job satisfaction among Lithuanian dentists. Ind Health. 2008;46:247-52.

9. Ahola K, Hakanen J. Job strain, burnout, and depressive symptoms: a prospective study among dentists. J Affect Disord. 2007;104:10310.

10. Kloostra PW, Eber RM, Inglehart MR. Anxiety, stress, depression, and patients' responses to periodontal treatment: periodontists' knowledge and professional behavior. J Periodontol. 2007;78:64-71.

11. Alemany Martínez A, Berini Aytés L, Gay Escoda C. The burnout syndrome and associated personality disturbances. The study in three graduate programs in Dentistry at the University of Barcelona. Med Oral Patol Oral Cir Bucal. 2008;13:E444-50.

12. Pöhlmann K, Jonas I, Ruf S, Harzer W. Stress, burnout and health in the clinical period of dental education. Eur J Dent Educ. 2005;9:78-84.

13. Gorter R, Freeman R, Hammen S, Murtomaa H, Blinkhorn A, Humphris G. Psychological stress and health in undergraduate dental students: fifth year outcomes compared with first year baseline results from five European dental schools. Eur J Dent Educ. 2008;12:61-8.

14. Happell B, Martin T, Pinikahana J. Burnout and job satisfaction: a comparative study of psychiatric nurses from forensic and a mainstream mental health service. Int J Ment Health Nurs. 2003;12:39-47.

15. Denton DA, Newton JT, Bower EJ. Occupational burnout and work engagement: a national survey of dentists in the United Kingdom. Br Dent J. 2008;205:E13.

16. Iacovides A, Fountoulakis KN, Moysidou C, Ierodiakonou C. Burnout in nursing staff: is there a relationship between depression and burnout? Int J Psychiatry Med. 1999;29:421-33.

17. Dormann C, Zapf D. Social stressors at work, irritation and depressive symptoms: accounting for unmeasured third variables in a multi-wave study. J Occup Organ Psychol. 2002;75:1.

18. Te Brake H, Bloemendal E, Hoogstraten J. Gender differences in burnout among Dutch dentists. Community Dent Oral Epidemiol. 2003;31:321-7.

19. Tselebis A, Moulou A, Ilias I. Burnout versus depression and sense of coherence: study of Greek nursing staff. Nurs Health Sci. 2001;3:69-71.

20. Carneiro SC, Vasconcelos BC, do Nascimento MM, Leal JL, Orestes MD, Porto GG. Occupational stress among Brazilian oral-maxillofacial surgeons. Med Oral Patol Oral Cir Bucal. 2009;14:e646-9. 